\title{
SAFE DESIGN AND OPERATION OF TANK REACTORS FOR MULTIPLE-REACTION NETWORKS: UNIQUENESS AND MULTIPLICITY
}

\author{
K. R. WESTERTER $P^{\dagger}$ and E. J. WESTERINK \\ Chemical Reaction Engineering Laboratories, Department of Chemical Engineering, Twente University of \\ Technology, PO Box 217, 7500 AE Enschede, Netherlands \\ (Accepted for puhlication 7 July 1989)
}

\begin{abstract}
A method is developed to design a tank reactor in which a network of reactions is carricd out. The network is a combination of parallel and consecutive reactions. The method ensures unique operation. Dimensionless groups are used which are either representative of properties of the reaction system or exclusively of the design and operating variables. In a plot of the optimal yield vs the dimensionless operating temperature the region is indicated where operation under conditions of uniqueness is feasible. The method is illustrated with an example: the air oxidation of benzene to maleic anhydride.
\end{abstract}

\section{INTRODUCTION}

The theory for multiple reactions in a tank reactor was elaborated by Westerterp (1962). Later Westerterp and Jansma (1985) demonstrated how a tank reactor can be designed and operated in the case when two simultaneous or consecutive first-order reactions are carried out. They showed that for a given yield or selectivity the possible region of operation can be derived from a plot of the dimensionless slope of the heat withdrawal line vs the dimensionless residence time. They accounted for the constraints to the operating and design conditions.

In their study, the dimensionless slope of the heat withdrawal rate (HWR) line was a function of the design and operating variables (i.e. residence time, feed concentration and an overall heat transfer parameter only). The constraints were a function of the design and operating variables as well as of the reaction system parameters. Hence, their constraints were of the form

$$
\frac{1+D a U^{*}}{\Delta \theta_{\mathrm{ad}}}>f\left(D a, S_{P} \text { or } \eta_{P}\right)
$$

or

$$
\frac{1+D a U^{*}}{\Delta \theta_{\mathrm{ad}}}<f\left(D a, S_{P} \text { or } \eta_{P}\right)
$$

For first-order reactions they demonstrated that in the case of consecutive reactions the selectivity is influenced by the temperature as well as by the residence time, while in the case of simultaneous reactions the selectivity is a function of the temperature only.

A different approach was presented by Balakotaiah and Luss (1984). They used catastrophe theory to investigate the entire parameter space for multiplicity. In their study the parameter space, consisting of operating and design variables, and kinetic parameters as well, was successively divided into subspaces

\footnotetext{
Author to whom correspondence should be addressed.
}

for which multiplicity was characterized. In the case of reaction networks their method leads to extensive analyses which are hard to relate to operating and design conditions. Therefore we will aim for a method that gives a good insight into all the relevant operating and design variables in a tank reactor in which a reaction network is executed.

In this study we will discuss a reaction network system combining both parallel and consecutive reactions. An infinite number of combinations is possible: we will limit ourselves to a network given by

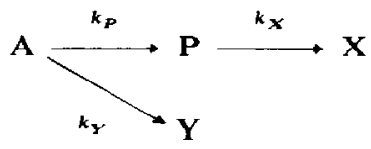

for which we regard the approach to this system as representative of all other networks. All reactions are irreversible and first-order, and have Arrhenius-type rate constants. We will discuss the design and operation of a tank reactor in which this particular reaction system is carried out and where $P$ is the desired product while $\mathrm{X}$ and $\mathrm{Y}$ are undesired by-products.

We must discriminate between reactor sections in which non-converted reactant can be recovered or not. In the case when recovery is possible the reactants are recycled to the inlet of the reactor; in that case the reactor should operate at high selectivities and low conversions so that the reactor design conditions chosen are mainly dominated by plant economics. This case will not be discussed here.

\section{DISCUSSION OF THE RELEVANT REACTION SYSTEM PARAMETERS}

Westerterp and Jansma (1985), Westerterp and Overtoom (1985), Westerterp and Ptasinsky (1984a, b) and Westerterp et al. (1984a) indicated that for two consecutive or two simultaneous reactions only the ratio of the activation energies $E_{X} / E_{P}=q$ is of interest for the choice of an adequate temperature regime (e.g. for $q>1$ low temperatures are required to obtain high 
yields). For reaction networks of more than two reactions such general statements are not possible anymore: each particular network requires an individual approach. For the network given in scheme (1) it is obvious that the case when both $E_{X}$ and $E_{Y}$ are lower than $E_{P}$ is not of any interest since in that case the reactor should operate at the highest possible temperature. This can be seen in Fig. 1(a). The reactor temperature in that case is limited by the mechanical and thermal properties of the construction material and of the catalyst only.

(a)

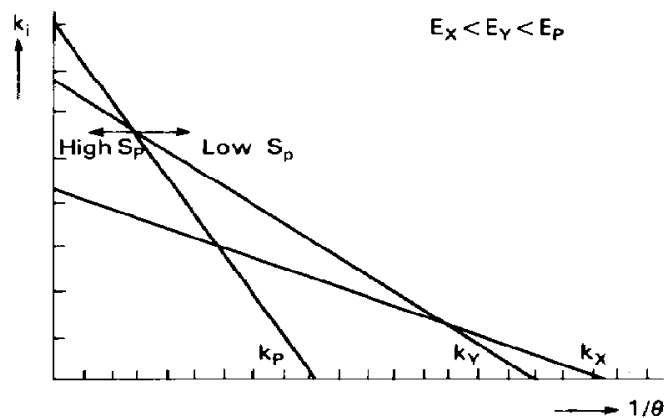

(b)

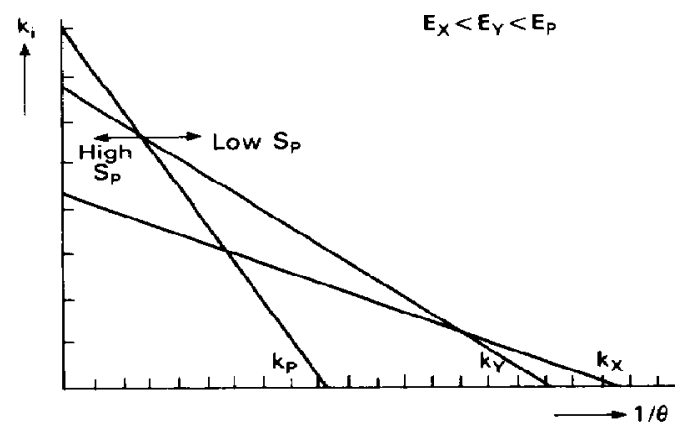

(c)

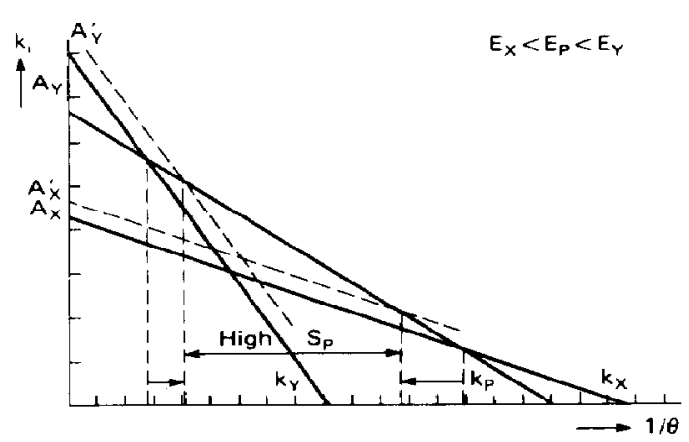

Fig. 1. (a) Arrhenius plot of a reaction system of three reactions with $E_{X}<E_{Y}<E_{P}$. (b) Arrhenius plot for a reaction system of three reactions with $E_{X}<E_{P}<E_{Y}$. (c) influence of the values of $A_{X}$ and $A_{Y}$ on the operating region.
Of more interest is a reaction system in which $E_{Y}$ is higher and $E_{X}$ is lower than $E_{r}$. A possible Arrhenius plot is given in Fig. 1(b). In principle we desire to operate as far as possible to the left-hand side in this plot because there temperatures and consequently also reaction rates are high. The selectivity is influenced by both the simultaneous and the consecutive reactions: at high temperatures $k_{\mathbf{r}} / k_{P}$ is high so in that case the selectivity is low; at low temperatures $k_{X} / k_{P}$ is high and conscquently the selectivity is low as well. Not only $p=E_{Y} / E_{P}$ and $q=E_{X} / E_{P}$ are relevant for our particular network but also the values of the preexponential constants $A_{X}, A_{Y}$ and $A_{P}$. These values too decide whether high yields are possible or not; they affect the region of temperatures that give a certain yield. As can be seen in Fig. 1(c), in the case when $A_{X}$ increases to $A_{X}^{\prime}$ the minimum required reactor temperature to obtain a chosen yield increases too. In the case when $A_{Y}$ increases to $A_{Y}^{\prime}$ the maximum allowable temperature to obtain a certain yield decreases. It can be concluded that it is impossible to achieve a yield of $100 \%$ since the consecutive reaction is favoured at low temperatures while the simultaneous reaction is favoured at high temperatures. Hence our network system must have a temperature and residence time at which the yield is at its maximum. We will aim for the maximum yield that is achievable within practical limits.

The method of deriving the relevant dimensionless groups has been discussed amply by Westerterp and Ptasinsky (1984), Westerterp and Jansma (1985) and Westerterp and Overtoom (1985), and will not be repeated here except for the method of deriving dimensionless rate constants for network systems. All groups are based on a reference reaction rate constant $k_{R}$ taken at a reference temperature $T_{R}$ : both are true constants based on the particular reaction system only. In our case at the chosen temperature $T_{R}$ the rate constant $k_{\mathrm{P}}$ equals the rate constant of the simultaneous reaction $k_{Y}$, hence

$$
k_{R}=k_{Y}\left(T_{R}\right)=k_{P}\left(T_{R}\right) \text {. }
$$

Using $k_{R}$ all reaction rate constants can be made dimensionless, according to

$$
k_{P} / k_{R}=\kappa=\exp \left[\gamma_{P}(1-1 / \theta)\right]
$$

with

and

$$
\begin{gathered}
\gamma_{P}=E_{P} / R T_{R} \text { and } \quad \theta=T / T_{R} \\
k_{Y} / k_{R}=\kappa^{p} \text { with } \quad p=E_{Y} / E_{P} \\
k_{X} / k_{R}=\beta \kappa^{q} \text { with } q=E_{X} / E_{P}
\end{gathered}
$$

$$
\beta=\frac{A_{X}}{A_{P}} \exp \left[\gamma_{P}(1-q)\right] .
$$

The heats of reaction can be made dimensionless by dividing the heats of reaction of the undesired reaction by that of the desired one, so

$$
H_{X}=\Delta H_{X} / \Delta H_{P} \text { and } H_{Y}=\Delta H_{Y} / \Delta H_{P} .
$$


The dimensionless groups, $p, q, \beta, \gamma_{P}, H_{X}$ and $H_{Y}$ fully describe the reaction system. They are independent of any design or operating variable.

\section{BASIC EQUATIONS}

We consider a tank reactor in which a combination of simultaneous and consecutive reactions according to scheme (1) occur. The reactions are first-order: the conversion rates are given by

$$
\begin{aligned}
& R_{w, A}=-\left(k_{P}+k_{Y}\right) C_{A} \\
& R_{w P}=k_{P} C_{A}-k_{X} C_{P} \\
& R_{w Y}=k_{Y} C_{A} \\
& k_{w X}=k_{X} C_{P}
\end{aligned}
$$

where $R_{w J}$ is expressed in moles converted of species $J$ per unit time and per unit catalyst mass.

We assume constant physical and thermochemical properties as well as constant coolant and reactor inlet temperatures. In our case the corresponding dimensionless mass and heat balances are

$$
\begin{aligned}
& X_{A}=\frac{D a\left(\kappa+\kappa^{p}\right)}{1+D a\left(\kappa+\kappa^{p}\right)} \\
& X_{P}=\frac{D a \kappa}{\left[1+D a\left(\kappa+\kappa^{p}\right)\right]\left(1+D a \beta \kappa^{q}\right)} \\
& X_{X}=D a \beta \kappa^{q} X_{P} \\
& X_{Y}=D a \kappa^{p}\left(1-X_{A}\right) \\
& \theta-\theta_{\mathbf{o}}=D a \Delta \theta_{\text {ad }}\left[\left(\kappa+H_{Y} \kappa^{p}\right)\left(1-X_{A}\right)+H_{X} \beta \kappa^{q} X_{P}\right] \\
&-D a U^{*}\left(\theta-\theta_{c}\right)
\end{aligned}
$$

where $D a, U^{*}, \theta_{c}$ and $\Delta \theta_{\text {ad }}$ are either operating or design variables representative of the residence time, the cooling power, the coolant temperature and the feed concentration of the key reactant. The relative conversions are interrelated by

$$
\begin{aligned}
\frac{C_{A o}-C_{A}}{C_{A o}} & =\frac{C_{P}}{C_{A o}}+\frac{C_{X}}{C_{A o}}+\frac{C_{Y}}{C_{A o}} \\
& =X_{A}=X_{P}+X_{X}+X_{Y}
\end{aligned}
$$

where $X_{P}$ is the yield realized at a given reactor temperature and a given residence time. The selectivity is defined as

$$
S_{P}=\frac{X_{P}}{X_{A}}=\eta_{P} / X_{A}
$$

Substitution of the relation for $X_{P}$ into eq. (7) and using the mass balance [eq. (2)] for $X_{A}$ to eliminate $D a$ gives

$$
S_{P}=\frac{\kappa}{\kappa+\kappa^{p}+\beta \kappa^{q} X_{A} /\left(1-X_{A}\right)} .
$$

In Fig. 2 the selectivity is plotted vs the conversion $X_{A}$ for several reactor temperatures. We see that for low temperatures the selectivity starts at values near $S_{P}=1$ and decreases rapidly: for higher temperatures the selectivity starts at values lower than 1 and decreases

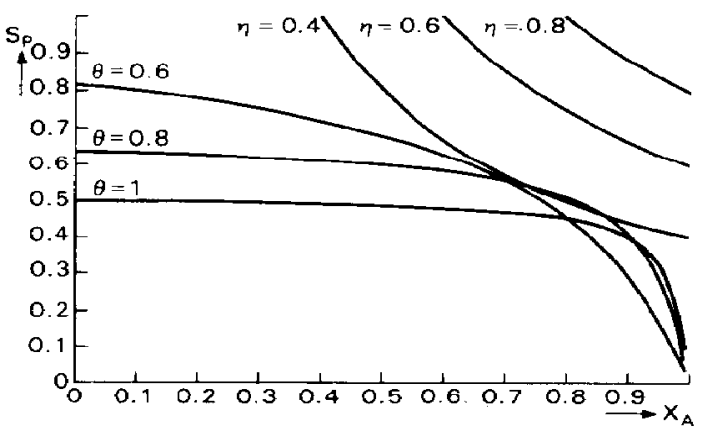

Fig. 2. Curves of selectivity vs conversion according to eq. (8) are given for several operating temperatures $(\theta=0.6,0.8$ and 1.0). Curves of constant yield $\eta=0.4,0.6$ and 0.8 according to the left-hand side of eq. (9) are also plotted. Data are $p=1.19$, $q=0.85, \gamma_{P}=14.9$ and $\beta=0.055$.

rather slowly until high conversions are reached. In Fig. 2 curves of constant yield $\eta_{P}$ are plotted: with increasing yield these hyperbolic curves shift to the right upper corner of the figure. As can be seen in Fig. 2 , for a given temperature lines of constant yield have either two, one or no points of intersection with the selectivity curves. In the case of two points of intersection the yield can be obtained at a high or a low degree of conversion. For curves with one point of intersection only one conversion is possible, while the given yield can never be obtained in the case of no points of intersection. In order to find the points of intersection we can set eq. (8) equal to eq. (7);

$$
S_{P}=\eta_{P} / X_{A}=\frac{\kappa}{\kappa+\kappa^{p}+\beta \kappa^{q} X_{A} /\left(1-X_{A}\right)} .
$$

After introduction of $S=1+\kappa^{p-1}$ and $R=\beta \kappa^{q-1}$ this leads to the quadratic equation

$$
X_{A}^{2}+\left(\eta_{P} R-\eta_{P} S-1\right) X_{A}+\eta_{P} S=0 .
$$

Since we investigate lines of constant yield we should beat in mind that in eq. (10) the value of $\eta_{P}$ is constant. The highest possible yield is reached in the case when only one solution exists. From the theory of quadratic equations we can derive that eq. (10) has one solution only in the case when

$$
\left(\eta_{P} R-\eta_{P} S-1\right)^{2}-4 \eta_{P} S=0 .
$$

Solving this equation for $\eta_{\max }$ leads to

$$
\eta_{\max }=\frac{1}{(\sqrt{R}+\sqrt{S})^{2}}=\frac{1}{\left(\sqrt{1+\kappa^{p-1}}+\sqrt{\beta \kappa^{q-1}}\right)^{2}} .
$$

Using eq. (11) the maximum achievable yield as a function of the reactor temperature can be calculated. In Fig. 3(a) the maximum yield vs the reactor temperature is plotted for a certain set of kinetic parameters. The corresponding values of the conversion $X_{A \max }$ are found by substitution of the yield $\eta_{\max }$ into the original quadratic equation eq. (10), so that the following equation is obtained:

$$
X_{A \max }=1-\sqrt{\eta_{\max } \beta \kappa^{q-1}} .
$$


(a)

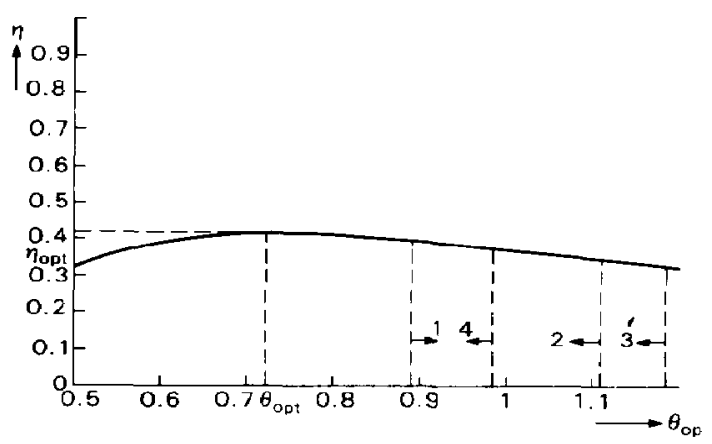

(c)

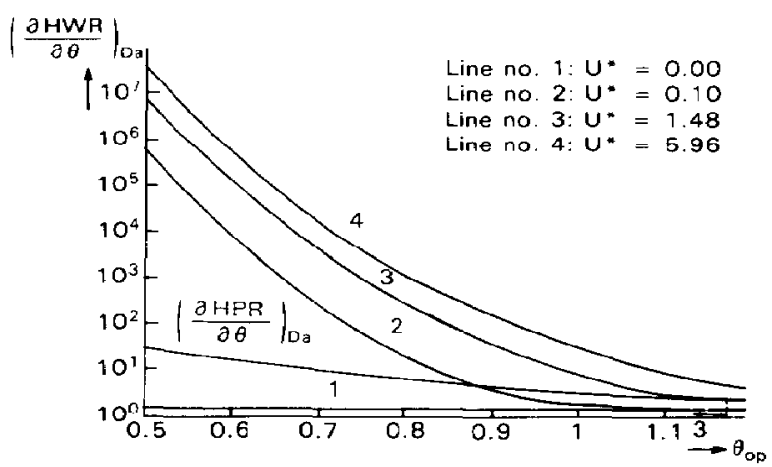

(b)

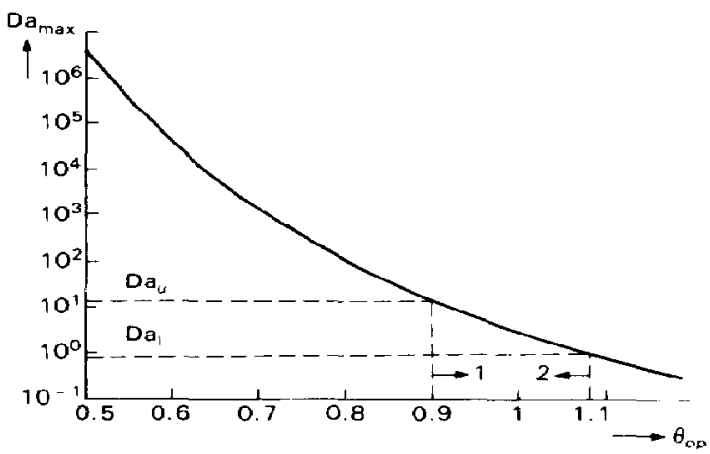

(d)

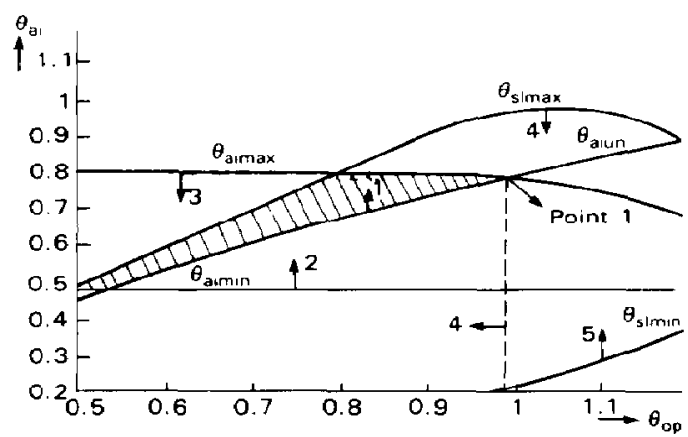

Fig. 3. (a) Maximum achievable yield vs the operating temperature. The vertical lines indicate the various constraints and their effect on the possible region of operating temperatures. Data are the same as for Fig. 2. (b) Dimensionless residence time $D a_{\max }$ vs the operating temperature $\theta_{00}$. Lines 1 and 2 indicate the range of allowable operating temperatures for practical values of the residence time. (c) Plot of the slopes of the HWR lines and HPR curves as a function of the operating temperature. The parameter of the curves of the HWR slope is $U^{*}$. Data are $p=1.19, q=0.85, \gamma_{P}=14.9, \beta=0.055, H_{X}=0.77, H_{Y}=1.77$ and $\Delta \theta_{\text {ad }}=0.63$. (d) Plot of the apparent feed inlet temperature $\theta_{a j}$ vs the operating temperature. Given are the curves corresponding to the maximum and minimum coolant tomperatures $\theta_{\text {aimax }}$ and $\theta_{\text {aimin }}$, and the required temperatures $\theta_{s i \max }$ and $\theta_{s i \max }$ calculated by means of condition ( $31 \mathrm{~b}$ ). $\theta_{\text {aiun }}$ is also plotted. See the text for explanation of the lines. Same data as in Fig. 3(c) plus $\theta_{c \max }=0.80, \theta_{\mathrm{cmin}}=0.48$ and $\theta_{o}=0.5$. Values for $U^{*}$ are $U_{\max }^{*}=5.96$ and $U_{\min }^{*}=0$.

According to relation (2) for $X_{A}$ the corresponding value of the residence time is

$$
D a_{\max }=\frac{1}{\kappa+\kappa^{p}} \frac{X_{A \max }}{1-X_{A \max }} .
$$

Once a reactor temperature is chosen, the reactor design is fixed by means of eqs (11)-(13), because we will desire to achieve the maximum yield that is possible at the given temperature.

As can be concluded from eq. (11) the maximum yield is still a function of the temperature. We will define the optimal yield $\eta_{\mathrm{opt}}$ as the maximum value of all maximum yiclds $\eta_{\max }$ The optimal temperature, at which the yield is optimal, is found by setting the derivative of eq. (11) with respect to the reactor temperature equal to zero. The following implicit equation for the optimal temperature is obtained:

$$
\frac{\beta(1-q)}{p-1}=\sqrt{\frac{\beta \kappa_{\mathrm{opt}}^{2 p-q}}{\kappa_{\mathrm{opt}}+\kappa_{\mathrm{opt}}^{p}}} .
$$

Again with eqs (11)-(13) the optimal reactor yield $\eta_{\text {opt }}$, conversion $X_{A \text { opt }}$ and residence time $D a_{\text {opt }}$ can be calculated.

Using the heat balance eq. (6) we are able to calculate the required values of the coolant $\theta_{c}$ and reactor inlet $\theta_{o}$ temperatures, and the required heat transfer number $U^{*}$ in order to operate at the required rcactor temperature. The heat balance eq. (6) can be written in terms of the heat production (HPR) and heat withdrawal (HWR) rates so that

$$
H P R=H W R .
$$

The HPR function, which is representative of the HPR, is given by

$$
\mathrm{HPR}=D a\left[\left(\kappa+H_{Y} \kappa^{p}\right)\left(1-X_{A}\right)+H_{X} \beta \kappa^{q} X_{P}\right] .
$$

The HWR function, which accounts for the heat withdrawal due to heat exchange with the cooling medium and to the heating up of the reactor feed, is 
given by

$$
\text { HWR }=\frac{1+D a U^{*}}{\Delta \theta_{\text {ad }}}\left(\theta-\theta_{a i}\right)
$$

where $\theta_{a i}$ is the apparent feed inlet temperature:

$$
\theta_{a i}=\theta_{o} \frac{1+D a U^{*} \theta_{c} / \theta_{o}}{1+D a U^{*}} \text {. }
$$

After elimination of $X_{P}$ and $X_{A}$, using eqs (2) and (3), the HPR term becomes

$$
\begin{aligned}
\text { HPR }= & D a\left\{\frac{\kappa+H_{Y} \kappa^{p}}{1+D a\left(\kappa+\kappa^{p}\right)}\right. \\
& \left.+\frac{D a H_{X} \beta \kappa^{q+1}}{\left[1+D a\left(\kappa+\kappa^{p}\right)\right]\left(1+D a \beta \kappa^{q}\right)}\right\} .
\end{aligned}
$$

For the operating points of the reactor the heat balance must hold, that is the HPR and HWR have to be equal. Hence, for a given operating point $\theta_{\mathrm{op}}$ :

$$
\operatorname{HPR}\left(\theta_{\mathrm{op}}\right)=\operatorname{HWR}\left(\theta_{\mathrm{op}}\right) \text {. }
$$

With eq. (18) the design and operating variables $U^{*}, \theta_{c}$ and $\theta_{c}$ can be calculated to ensure operation at $\theta_{\mathrm{op}}$ and $D a_{\max }$, where $D a_{\max }$ is calculated by means of eq. (13).

\section{CONSTRAINTS TO THE DESIGN AND OPERATING CONDITIONS}

The conditions in a tank reactor cannot be chosen at will as was already mentioncd by Wcsterterp and Jansma (1985). The conditions are limited because of stability and uniqueness requirements as well as practical limits due to the reactor lay-out and operation. The various constraints now will be discussed and demonstrated. For each constraint [see Fig. 3(b)-(d)] the possible temperature region is determined by projecting the limits of each constraint on the temperature axis of a plot where the maximum yield is plotted for each operating temperature [see Fig. 3(a)]. These constraints can be presented in one plot as well [see Fig. 7]. This was demonstrated by Westerterp and Jansma (1985), who showed that all constraints could be indicated in a plot of the slope of the heat withdrawal line vs the dimensionless residence time. For each constraint the corresponding boundary limits are plotted in Fig. 7.

\section{Constraints on the residence time}

The possible region for the residence time $\tau$ is mainly dominated by the reactor volume that can be installed and well-mixed, and by the time required for reaching steady-state conditions after start-up or operational changes. For liquid-phase reactions in a tank reactor, i.e. $\tau$ is usually between 500 and $10,000 \mathrm{~s}$ while for gas-solid fluid beds $\tau$ is hetween 1 and $10 \mathrm{~s}$. However, these limits are not sharp. The dimensionless residence time $D a$ is related to the physical residence time according to

$$
\tau=D a / k_{R} \rho_{b} .
$$

With these data it is possible to calculate the upper and lower limit of the dimensionless residence time, $D a_{u}$ and $D a_{1}$.

For each operating temperature $\theta_{\text {op }}$ the residence time $D a_{\max }$ required to reach maximum yield is calculated. Using eq. (13) after elimination of $\eta_{\max }$ and $X_{A \max }$ by means of eqs (11) and (12) the following relation is obtained:

$$
D a_{\max }=\frac{1}{\kappa+\kappa^{p}} \sqrt{\frac{\kappa+\kappa^{p}}{\beta \kappa^{q}}} .
$$

In eq. (19) $D a_{\max }$ is only dependent on the reaction temperature. Possible values of $D a_{\max }$ should be between $D a_{u}$ and $D a_{l}$. Hence $D a_{u}$ and $D a_{l}$ also set an upper and lower limit to the operating temperature. How these constraints work out is shown in Fig. 3(b). The possible region of operating temperatures that remains after these two constraints are fulfilled is indicated in Fig. 3(b): the boundaries are given by lines 1 and 2 . Using the method presented by Westerterp and Jansma (1985) these constraints lead to lines 1 and 2 in Fig. 7.

Constraints on the cooling area in relation to stability

The dimensionless group representative of the cooling capacity is $U^{*}=U A /\left(\rho_{b} k_{R} \rho_{g} C_{p g} V_{R}\right)$. The lower limit of the cooling capacity is given by $U^{*}=0$ : in that case the reactor operates under adiabatic conditions. The upper limit $U_{\max }^{*}$ is given by the maximum cooling capacity that can be installed: it is dominated by the ratio $U A / V_{R}$. This ratio depends on the overall heat transfer coefficient $U$ and the maximum amount of cooling area that can be installed per unit of reactor volume. The latter depends on the layout of the reactor, i.e. whether an internal or external cooler is installed. For an operating point to be stable the slope of the heat withdrawal line must be higher than the slope of the heat production curve at the operating temperature. For a given reactor design $D a$ is constant so that, after partial differentiation at constant $D a$ of both heat terms, we find the following condition:

$$
\frac{1+D a U^{*}}{\Delta \theta_{\mathrm{ad}}}>\left(\frac{\partial \mathrm{HPR}}{\partial \theta}\right)_{D a_{\max }} \text { at } \theta_{\mathrm{op}}
$$

where $\theta_{\mathrm{op}}$ is the operating temperature, and $D a_{\max }$ the residence time required to achieve maximum yield. $D a_{\max }$ is calculated using eq. (19).

We see that the left-hand side of this criterion depends on the operating and design variables $U^{*}$ and $\Delta \theta_{\text {ad }}$. It can be concluded that the reactor will operate in a stable state at high values of $U^{*}$ and low values of $\theta_{\mathrm{ad}}$, so that the stability of the reactor strongly depends on the ratio $U A / V_{R}$ and the feed inlet concentration $C_{A o}$, since $\Delta \theta_{\mathrm{ad}}=-\Delta H_{P} C_{A o} / T_{R} \rho_{g} C_{p g}$. In practice there are certain limits to the feed concentration, because for economical reasons feed dilution will be avoided and, for example, for safety reasons the reactor must operate outside the explosion limits in the case of oxidation reactions. Due to these restric- 
tions on $\Delta \theta_{\text {ad }}$ the ratio $U A / V_{R}$ dominates. In Fig. 3(c) the slope of the heat production curve is plotted vs the operating temperature. For some selected values of $U^{*}$ the slope of the heat withdrawal line is plotted as well. Only temperatures to the left of the points of intersection are available for stable operation, because there eq. (20) is fulfilled.

The effect of the stability requirement on the region of operating temperatures is shown in Fig. 3(c). For a given value of $U^{*}=1.48$ operation is possible to the left of the point of intersection of both lines, indicated by line 3 .

In Fig. 7 the stability requirement is given by line 7 , which represents the slope of the heat production curve $\partial \mathrm{HPR} / \partial \theta$ in the operating point at $D a=D a_{\max }$ We now must choose our operating conditions above line 7.

\section{The uniqueness requirement}

The design engineer and the operator in general desire to avoid the situation of multiple steady states or multiplicity; they aim for uniqueness, that is one stable operating point only. There is no multiplicity if $\operatorname{HWR}\left(\theta_{\mathrm{op}}\right)=\operatorname{HPR}\left(\theta_{\mathrm{op}}\right)$ is the only solution to HPR $=$ HWR. There are many ways to determine the parameter values that lead to unique operation. Well known is the use of catastrophe theory as elaborated by Balakotaiah and Luss (1984). Although we use different parameter groups the method presented below is equivalent to the one presented by Balakotaiah and Luss. The critical parameter values required for our method can be obtained along the lines presented here. In our case theoretically up to seven operating points are possible, four stable and three unstable ones. This is shown in Fig. 4. In order to find the boundary between uniqueness and multiplicity we define a function $\mathrm{F}(\theta)$ as

$$
F(\theta)=\frac{\operatorname{HWR}(\theta)}{\operatorname{HPR}(\theta)}-1
$$

For an operating point $\theta_{\text {op }}$ this function must equal to zero; a typical plot of $F(\theta)$ vs $\theta$ is given in Fig. 5, in which three possible operating points can be distinguished.

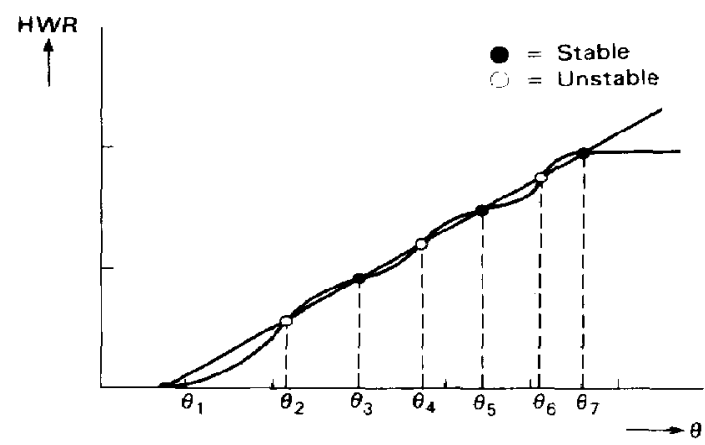

Fig. 4. Illustration of the case of multiple operating points. Each intersection of the HPR curve and the HWR line is a possible operating point.

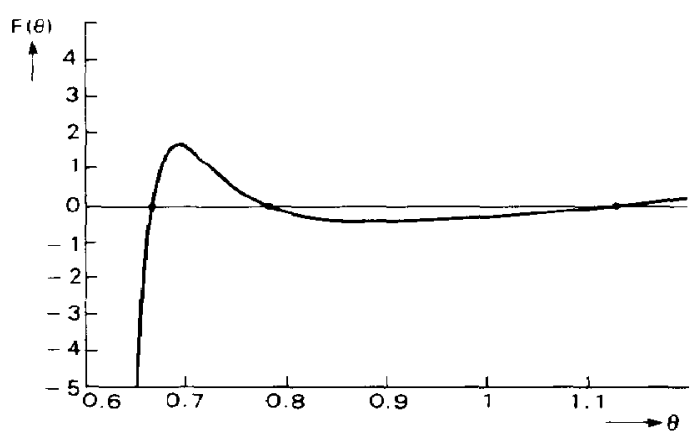

Fig. 5. $F(\theta)=$ HWR $/ H P R-1$ vs the reactor temperature.

Now if the slope of the HWR line passing through the point $\operatorname{HPR}\left(\theta_{\mathrm{op}}\right), \theta_{\mathrm{op}}$ is diminished, eventually a value of the slope is reached where the HWR line touches the HPR curve. This is illustrated in Fig. 6. At this point two operating points are obtained and $\theta_{a i}$ reaches $\theta_{a i u n}$. As can be seen, the requirement depends on the value of the both $U^{*}$ and $\theta_{a i}$. In this situation for eq. 20 we can state that the $F(\theta)$ curve only touches the horizontal line $F(\theta)=0$ at a point distinct from $\theta_{\text {op }}$, say at $\theta_{j}$. We developed the following procedure to find the values of $\theta_{j}$. If $F(\theta)$ touches the line $F(\theta)=0$ at $\theta_{j}$ the following relations hold:

$$
\begin{gathered}
\operatorname{HWR}\left(\theta_{\mathrm{op}}\right)=\frac{1+D a U^{*}}{\Delta \theta_{\mathrm{ad}}}\left(\theta_{\mathrm{op}}-\theta_{a i}\right) \\
\operatorname{HWR}\left(\theta_{j}\right)=\frac{1+D a U^{*}}{\Delta \theta_{\mathrm{ad}}}\left(\theta_{j}-\theta_{a i}\right) \\
{\left.\left[\frac{\partial F(\theta)}{\partial \theta}\right]_{D a}\right|_{\theta_{j}}=0 .}
\end{gathered}
$$

From eq. (24) we have

$$
\operatorname{HPR}\left(\theta_{j}\right) \operatorname{HWR}^{\prime}\left(\theta_{j}\right)-\operatorname{HPR}^{\prime}\left(\theta_{j}\right) \operatorname{HWR}\left(\theta_{j}\right)=0
$$

where

$$
\begin{aligned}
\operatorname{HWR}^{\prime}\left(\theta_{j}\right) & =\left(\frac{\partial \mathrm{IWR}}{\partial \theta}\right)_{D a} \text { and } \\
& \times \operatorname{HPR}^{\prime}\left(\theta_{j}\right)=\left(\frac{\partial \mathrm{HPR}}{\partial \theta}\right)_{D a} .
\end{aligned}
$$

Since $\operatorname{HPR}\left(\theta_{j}\right) \neq 0$ and $\operatorname{HWR}\left(\theta_{j}\right) \neq 0$ we have

$$
\frac{\operatorname{HPR}^{\prime}\left(\theta_{j}\right)}{\operatorname{HPR}\left(\theta_{j}\right)}-\frac{\operatorname{HWR}^{\prime}\left(\theta_{j}\right)}{\operatorname{HWR}\left(\theta_{j}\right)}=0 .
$$

From the definition of HWR we have

$$
\operatorname{HWR}^{\prime}\left(\theta_{j}\right) / \operatorname{HWR}\left(\theta_{j}\right)=\left(\theta_{j}-\theta_{a i}\right)^{-1} \text {. }
$$

Substitution into eq. (26) yields

$$
\frac{\operatorname{HPR}\left(\theta_{j}\right)}{\operatorname{HPR}^{\prime}\left(\theta_{j}\right)}=\theta_{j}-\theta_{a i}
$$

From eqs (22) and (23) we have

$$
\theta_{a i j}=\frac{\theta_{\mathrm{op}} \operatorname{HPR}\left(\theta_{j}\right)-\theta_{j} \operatorname{HPR}\left(\theta_{\mathrm{op}}\right)}{\operatorname{HPR}\left(\theta_{j}\right)-\operatorname{HPR}\left(\theta_{\mathrm{op}}\right)} .
$$


In eq. (28) the index aij refers to the apparent feed inlet temperature corresponding to the conditions where $\theta_{f}$ is just an operating point.

Substitution of this equation into eq. (27) leads to the following implicit equation:

$\operatorname{HPR}\left(\theta_{j}\right)-\operatorname{HPR}\left(\theta_{\mathrm{op}}\right)+\left(\theta_{\mathrm{op}}-\theta_{j}\right) \operatorname{HPR}^{\prime}\left(\theta_{j}\right)=0$

Equation (29) can have multiple solutions for $\theta_{j}$ which are found using trial-and-error techniques. We searched the $\theta_{j}$-axis for given values of $\theta_{\mathrm{op}}$ and $D a_{\max }$ In theory the entire $\theta_{j}$-axis should be scanned for solutions: in practice the region $0.25 \theta_{\mathrm{op}}<\theta_{j}<2 \theta_{\mathrm{op}}$ was sufficient. Calculating $\theta_{a i j}$ from eq. (28) and taking the highest value of $\theta_{a i j}$ gives the value of $\theta_{a i u n}$, above which the reactor is operating at conditions of uniqueness.

Since $\theta_{a i}$ is affected by the operating and design variables $U^{*}$ and $\theta_{v} / \theta_{v}$ it can be understood that $U^{*}$ and $\theta_{c} / \theta_{o}$ will have a great influence on the uniqueness requirement. Or these variables the feed inlet temperature $\theta_{o}$ is normally taken as the temperature at which the feed is available.

There are three conditions which must be satisfied. Firstly, the value chosen for $\theta_{a i}$ under operating conditions must be higher than $\theta_{\text {aiun }}$ to avoid multiplicity. Hence

$$
\theta_{a i}>\theta_{a i u n}
$$

or using the heat balance eq. (18):

$$
\frac{1+D a U^{*}}{\Delta \theta_{\text {ad }}}>\frac{\operatorname{HPR}\left(\theta_{\text {op }}\right)}{\theta_{\text {op }}-\theta_{\text {aiun }}} \text {. }
$$

Secondly, the slope of the heat withdrawal line required to operate under unique conditions must be lower than the maximum slope and higher than the minimum slope that can be achieved in our particular reactor. This leads to the conditions for the reactor with optimal residence time and maximum cooling area:

$$
\frac{\operatorname{HPR}\left(\theta_{\mathrm{op}}\right)}{\theta_{\mathrm{op}}-\theta_{a \mathrm{i}}}<\frac{1+D a_{\max } U_{\max }^{*}}{\Delta \theta_{\mathrm{ad}}}
$$

and for the adiabatic reactor:

$$
\frac{\operatorname{HPR}\left(\theta_{\mathrm{op}}\right)}{\theta_{\mathrm{op}}-\theta_{a i}}>\frac{1}{\Delta \theta_{\mathrm{ad}}} \text {. }
$$

These conditions can be rearranged to

$$
0_{a i}=\theta_{a l \max }<\left[\theta_{\mathrm{op}}-\frac{\Delta \theta_{\mathrm{ad}} \operatorname{HPR}\left(\theta_{\mathrm{op}}\right)}{1+D a_{\max } U_{\max }^{*}}\right]
$$

and

or also

$$
\theta_{a i}=\theta_{s t m i n}>\left[\theta_{\mathrm{op}}-\Delta \theta_{\mathrm{ad}} \operatorname{HPR}\left(\theta_{\mathrm{op}}\right)\right]
$$

$$
\theta_{s i \min }<\theta_{a i}<\theta_{s i \max } .
$$

The right-hand side of conditions $31(\mathrm{~b})$ is referred to as $\theta_{s l}$, that is the value of $\theta_{a i}$ for the maximum or the minimum value of $U^{*}$. The right-hand side of conditions 31(a) correspond to the minimum or maximum value for the slope of the heat withdrawal line through the operating point $\theta_{\text {op }}, \operatorname{HPR}\left(\theta_{\mathrm{op}}\right)$ The two values of $\theta_{s 1}$ are shown in Fig. 6.

Thirdly, there are practical limits to the temperature of the coolant. The possible values of $\theta_{c}$ depend on the choice of the coolant, e.g. the following temperature region is possible in the case of boiling water, $180-320^{\circ} \mathrm{C}$, for oils $200-400^{\circ} \mathrm{C}$ or for molten salts $150-500^{\circ} \mathrm{C}$. Each coolant chosen leads to a maximum and a minimum value of $\theta_{a i}$, so for $\theta_{a i}$ the following condition holds:

$$
\theta_{a i \min }<\theta_{a i}<\theta_{a i \max }
$$

or using the heat balance eq. (18):

$$
\frac{\operatorname{HPR}\left(\theta_{\mathrm{op}}\right)}{\theta_{\mathrm{op}}-\theta_{\text {aimin }}}<\frac{1+\operatorname{Da} U^{*}}{\Delta \theta_{\mathrm{ad}}}<\frac{\operatorname{HPR}\left(\theta_{\mathrm{op}}\right)}{\theta_{\mathrm{op}}-\theta_{\text {aimax }}}
$$

Figure 3(d) shows how the criteria work out, if $\theta_{a i}$ vs $\theta_{\text {op }}$ is plotted. In the diagram the significance of conditions (30a), (31b) and (32a) is demonstrated. Line 1 gives the values of $\theta_{\text {aiun }}$, line 2 gives the values of $\theta_{\text {aimin }}$, line 3 values of $\theta_{\text {aimax }}$, line 4 shows $\theta_{\text {slmax }}$ and line 5 shows the values of $\theta_{\text {simin }}$. When all constraints are satisfied an area of possible $\theta_{a i}$ values remains. In this area unique operation is possible for certain values of $\theta_{a t}$. We can see that unique conditions are possible for a limited region of $\theta_{\text {op }}$ values, in our case the shaded area to the left of point 1.

In Fig. 7 the constraints of the coolant temperature as calculated with cq. (17) and condition (32b) are accounted for by lines 5 and 6 ; we should operate above line 5 and below line 6 . The constraints on the cooling area available, as given by conditions (31b), are given as lines $3\left(U^{*}=0\right)$ and $4\left(U^{*}=U_{\max }\right)$. Operation is possible above line 3 and below line 4 . The uniqueness requirement as calculated with eqs (28) and (29) and with condition (30b) is given by line 8 . Safe operation therefore is possible above line 8 .

\section{POSSIBLE REGION OF OPERATION}

The effect of the various constraints on the operating temperature is indicated in Fig. 3(a). For every

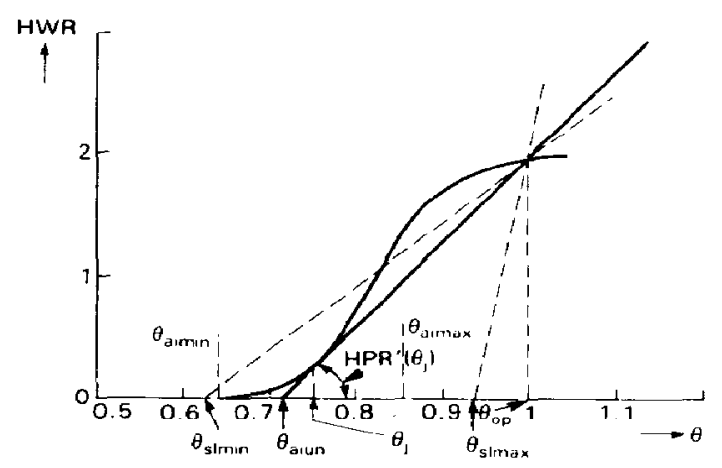

Fig. 6. Illustration of the method used to determine the lowest value of the apparent reactor inlet temperature $\theta_{\text {aiur }}$ above which the reactor operates under conditions of uniqueness. 


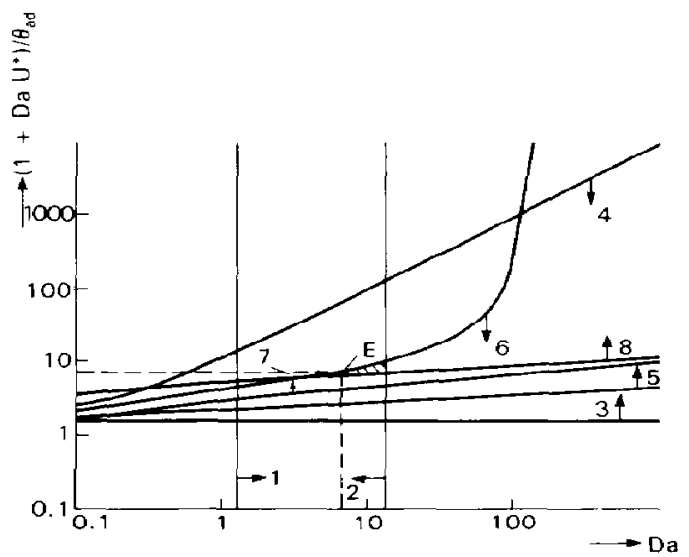

Fig. 7. Area of operation for the oxidation of benzene to maleic anhydride. Data are the same as for Fig. 3. The lines indicate the restrictions: lines 1 and 2 due to the residence time, lines 3 and 4 due to the cooling areas, lines 5 and 6 due to the coolant temperatures, line 7 accounts for the stability requirement, and line 8 for the uniqueness requirement. The arrow indicate the regions where operation is possible according to the restriction under consideration

constraint the possible region of operating temperatures is projected on the $\theta_{o p}$-axis. Lines 1 and 2 indicate the constraints due to the residence time, line 3 is due to the stability requirement, and line 4 is due to the maximum coolant temperature. Taking all constraints into account the possible range of temperatures is reduced to a small region between lines 1 and 4 [see Fig. 3(a)]. As can be seen the optimum yield is obtained outside the possible region of operation. The highest yield that can be achieved is located on the boundary of the region. Using this plot a temperature can be selected in order to design a reactor. To keep sufficient flexibility the temperature should not be chosen at the boundary, since adaption of the operating temperature to changes in residence time should be possible in order to be able to operate at varying reactor loads.

In Fig. 7 the area of operation that remains when all constraints are satisfied can be indicated as well. The possible area has been shaded; in this area safe unique operation is possible.

\section{DESIGN PROCEDURE FOR A TANK REACTOR IN WHICH MULTIPLE REACTIONS OCCUR AND WHICH OPERATES SAFELY UNDER UNIQUE CONDITIONS}

We will discuss a possible procedure for designing a tank reactor where the maximum yield has to be aimed at within the possible region of operation. The procedure could go along the following lines:

(1) Make an Arrhenius plot of all reaction rate constants vs the reciprocal temperature. Determine whether operating temperatures required to obtain high yields have to be high, low or somewhere in an intermediate region.
(2) Determine for the reaction system under consideration the proper values of $k_{R}, T_{K}, p, q, \beta, \gamma_{P}$, $H_{X}$ and $H_{Y}$.

(3) Draw a diagram like Fig. 3(a). Determine from practical considerations the allowable values of the residence time $\tau=V_{R} / \Phi$ and calculate the limits $D a_{u}$ and $D a_{1}$ according to

$$
D a=k_{R} \rho_{b} \tau \text {. }
$$

Calculate the temperature limits $T_{u}$ and $T_{I}$ solving eq. (19) for $D a_{\omega}$ and $D a_{l}$. Now calculate the corresponding yields $\eta_{u}$ and $\eta_{l}$ using eq- (11).

(4) Calculate the optimal temperature $\theta_{\text {opt }}$, solving the implicit eq. (14) and the corresponding value of $D a_{\text {opt }}$ using eq. (19). If $\theta_{\text {opt }}$ lies within the range of possible temperatures as determined in step 3 $\theta_{\text {opt }}$ should be chosen as the design value for the operating temperature. Otherwise an operating temperature close to either $\theta_{l}$ or $\theta_{u}$ should be chosen. In the case when the values of the yield $\eta_{u}$ or $\eta_{l}$ are too low the reaction cannot be executed economically in a tank reactor without the recycle of non-converted reactant.

(5) Calculate possible values of $\Delta \theta_{\mathrm{ad}}$, taking into account explosion limits and available feed concentrations.

(6) Draw a diagram like Fig. 7 and determine under which combinations of values of $D a, U^{*}$ and $\Delta \theta_{\text {ad }}$ safe and unique operation is feasible. From these data the corresponding values of $\theta_{\mathrm{op}}$ and $\eta_{P}$ can be calculated.

(7) Choose the design value of $\theta_{\text {op }}$ that ensures the highest possible yield within the range of possible temperatures. An operating temperature can be selected from Fig. 3(a). The design engineer should be aware of the fact that changes in reactor loads must be possible. To obtain sufficient flexibility the operating temperature should not be chosen on the boundary of the possible temperature region. The effect of changes in reactor loads can be checked in Fig. 7.

(8) Complete the design by calculating the design values of $D a, U^{*}$ and $\theta_{a i}$, and from $\theta_{a i}$ the value of $\theta_{c}$ corresponding to economical feed inlet temperatures $\theta_{0}$.

This procedure will now be demonstrated in an example.

\section{AN EXAMPLE}

The applicability of the method outlined will be demonstrated for the air oxidation of benzene to maleic anhydride. This product can be made in a fluidized-bed reactor. We, of course, realise ourselves that a fluid-bed reactor does not behave as a tank reactor. For the moment we will consider it as a possible approximation of the tank reactor. In the following paper we discuss the fluid-bed reactor directly and in more detail. The reaction scheme 
as presented by Wohlfahrt and Emig (1980) consists of three reactions:

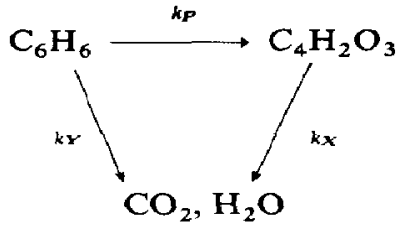

All reactions are considered to be first-order in the reactant, while an excess of oxygen is applied. Furthermore we assume the maximum cooling capacity that can be installed to be $8000 \mathrm{~W} / \mathrm{K} \mathrm{m}^{3}$ and the feed concentration to be 1 mole \% benzene. From Wohlfahrt and Emig we learn that

$$
\begin{aligned}
& k_{P}=4280 \exp (-12,660 / T) \mathrm{m}^{3} / \mathrm{kg} \text { catalyst s } \\
& k_{Y}=70,100 \exp (-15,031 / T) \mathrm{m}^{3} / \mathrm{kg} \text { catalyst s } \\
& k_{X}=26 \exp (-10,794 / T) \mathrm{m}^{3} / \mathrm{kg} \text { catalyst s }
\end{aligned}
$$

Furthermore the following physical data are available:

$$
\begin{aligned}
C_{p g} & =1.09 \mathrm{~kJ} /(\mathrm{kg} \mathrm{K}) \\
\rho_{g} & =1.01 \mathrm{~kg} / \mathrm{m}^{3} \\
\rho_{b} & =650 \mathrm{~kg} / \mathrm{m}^{3} \\
C_{A D} & =1.0 \mathrm{~mole} \% .
\end{aligned}
$$

The following values for the parameters are obtained:

$$
\begin{gathered}
T_{R}=848 \mathrm{~K}, \quad \gamma_{P}=14.9, \quad q=0.85, \\
H_{X}=0.77, \quad \Delta \theta_{\mathrm{ad}}=0.63, \\
k_{R}=1.4 \times 10^{-3} \mathrm{~m}^{3} /(\mathrm{kg} \mathrm{s}), \\
\beta=0.55, \quad p=1.19, \quad H_{Y}=1.77 .
\end{gathered}
$$

With these data a plot of the maximum yield versus the reactor temperature can be calculated: it is given in Fig. 3(a). As can be seen an optimal yield of only $43 \%$ can be achieved. The curve is very smooth so the maximum yield is not very sensitive towards changes in temperature. This follows from the values of $p$ and $q$ being rather near 1.00 .

In Fig. 3(b) the required values of $D a$ are plotted vs the reactor temperature: both limits $D a_{u}$ and $D a_{i}$ are projected on the $\theta$-axis. As can be seen the required reactor tempertures are much higher than the optimal temperature $\left(A_{\mathrm{opt}}=0.72\right)$ : this is due to the fact that the reaction rates are too low at this optimal temperature. As can be seen in Fig. 3(a) the maximum achievable yield is about $40 \%$. In Fig. $3(\mathrm{c})$ the derivative of the HPR curve at operating conditions ( $D a_{\max }$ and $\left.\theta_{\mathrm{op}}\right)$ is plotted as well as four slopes of the HWR line for $U^{*}$ $=5.96,1.48,0.1$ and 0 for $D a=D a_{\max }$. As can be concluded from the projection on the $\theta$-axis the stability requirement has effect on the operating area only in the case when the values of $U^{*}$ are small. In that case the reactor operates almost adiabatically. In Fig. 3(d) the values of $O_{\text {aiun }}$ for each operating point are plotted as well as the two constraints on the apparent feed inlet temperature $\left(\theta_{a i \min }\right.$ and $\left.\theta_{a i \max }\right)$. Of course $\theta_{a i}$ should be higher than $O_{a i u n}$ to ensure unique oper- ation. On the other hand $\theta_{a j}$ should be lower than $\theta_{s t \max }$ otherwise the required cooling area cannot be installed in the reactor. For this example we have assumed our coolant to be a molten salt operating in the temperature range of $140-400^{\circ} \mathrm{C}$. All constraints can be projected on the $\theta_{\text {op }}$-axis in Fig. 3(a). Taking all constraints into account leads us to the possible range of operating temperatures of $0.90<\theta_{\mathrm{op}}<0.98$. Hence, operation is limited by the maximum residence time and by the maximum coolant temperature. In Fig. 7 the region of possible operating conditions is given by the shaded area: again from Fig. 7 we can see that operation is limited by the longest possible residence time and the maximum coolant temperature. The region of feasible operation is rather small. As the design conditions we now take point $E$ in Fig. 7 with $D a=8$ and $\left(1+D a U^{*}\right) / \Delta \theta_{\mathrm{ad}}=7.5$. Here we operate at $T_{\mathrm{op}}=794 \mathrm{~K}$ and with $C_{A o}=0.01$ mole fraction of benzene. From the shaded area and as outlined by Westerterp and Jansma (1985) we now can determine that the reactor load can be changed from 80 to $160 \%$ of the design value and the concentration from 0.88 to 1.07 mole \%. The possible operating range is rather narrow. Taking into account this rather limited ability to adapt the operating conditions to changes in reactor load we may conclude that it is hardly possible to carry out the oxidation of benzene to maleic anhydride in a tank reactor or other well-mixed reactors. In practice these reactions are carried out in a tabular reactor.

\section{CONCLUSIONS}

For multiple-reaction networks executed in a tank reactor we presented a method to design a reactor for safe operation at high yields. With respect to the method we may conclude that:

(1) Each reaction network requires an individual approach: the optimal temperature policy strongly depends on the kinetic parameters of all the reactions involved.

(2) Kinetic formation is required about all reactions, both the desired and undesired ones.

(3) A design method is obtained that ensures a maximum yield within the possible range of operating conditions.

(4) For the network given it is not possible to achieve $100 \%$ yield.

(5) It gives good insight into the effect of changes of operating variables.

(6) In contrast to the approach of Balakotaiah and Luss (1984) in which many cross sections through a multi-dimensional space are required to present their results, we only need a twodimensional plane to provide all relevant information for the design and operation of a tank reactor for one particular reaction network. This is due to our rigorously separating dimensionless groups in those containing reaction 
system variables and those with design and operating variables, and moreover by inclusion of only one single variable in each dimensionless number [see Westerterp and Jansma (1985)].

We have to realize that kinetic data in practice may deviate from those used in the design. One may adjust for this discrepancy by executing a test run after startup of the reactor section. With the adjusted parameter values the model enables us to obtain quickly the best set of operating conditions.

$A$
$C_{J}$
$C_{p g}$
$D a$
$E$
$H_{i}$
$-\Delta H_{P}$
$k$

$k_{R}$
$p$
$q$
$R_{w}$
$S_{P}$
$T$
$T_{R}$
$U$
$U *$
$V_{R}$
$X$

\section{NOTATION}

pre-exponential factor, $\mathrm{m}^{3} /(\mathrm{kg} \mathrm{s})$

concentration of species $J, \mathrm{~mol} / \mathrm{m}^{3}$

specific heat of the reaction mixture, $J /(\mathrm{kg} \mathrm{K})$

$k_{R} \rho_{b} V_{R} / \Phi$, dimensionless residence time

$E \quad$ activation energy, $\mathrm{J} / \mathrm{mol}$

$H_{i} \quad \Delta H_{i} / \Delta H_{p}$, ratio of reaction heats

$-\Delta H_{P}$ heat of reaction, $\mathbf{J} / \mathrm{mol}$

reaction rate constant $\left[k_{i}=A_{i} \exp \left(-E_{i}\right)\right.$

$R T)], \mathrm{m}^{3} /(\mathrm{kg} \mathrm{s})$

$k_{R} \quad$ reference reaction rate constant, $\mathrm{m}^{3} /(\mathrm{kg} \mathrm{s})$

$\quad E_{Y} / E_{P}$

$q \quad E_{X} / E_{P}$

$R_{w} \quad$ rate of production of species, $\mathrm{mol} /(\mathrm{kg} \mathrm{s})$

$S_{P} \quad$ integral selectivity

$T$ temperaturc, $\mathrm{K}$

$T_{R} \quad$ reference temperature, $\mathrm{K}$

$U$ total heat transfer coefficient, $W /\left(\mathrm{m}^{2} \mathrm{~K}\right)$

$U A / k_{R} \rho_{b} \rho_{g} C_{p g} V_{R}$

$\boldsymbol{X}$ volume of the reactor, $\mathrm{m}^{3}$ relative degree of conversion

Greek letters

$\beta \quad$ pre-exponential factor, $\mathrm{m}^{3} /(\mathrm{kgs})$

$\gamma_{P}$ dimensionless activation temperature $\left(E_{P} / R T_{R}\right)$

$\eta \quad$ yield, moles of $P$ produced divided by the number of moles that would be produced if all reactant $A$ were converted into $P$

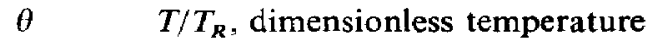

$\Delta \theta_{\mathrm{ad}} \quad-\Delta H_{P} C_{A o} / T_{\mathrm{R}} \rho_{g} C_{p g}$, dimensionless adiabatic temperature rise

$\kappa \quad k_{P} / k_{R}$, dimensionless rate constant

$\rho_{b} \quad$ bulk density of the catalyst, $\mathrm{kg} / \mathrm{m}^{3}$ $\rho_{g}$ density of the reaction mixture, $\mathrm{kg} / \mathrm{m}^{3}$

(I) gas load, $\mathrm{m}^{3} / \mathrm{s}$

Subscripts

A reactant

ai apparent feed inlet

aiun apparent feed inlet at which just uniqueness is achieved

aij apparent feed inlet at which the number of multiple operating points is just reduced by one

c coolant

$\max$ maximum at given temperature

$o \quad$ inlet conditions

op at operating conditions

opt absolute optimum

$P \quad$ product

$X \quad$ undesired product formed by the consecutive reaction

$Y$ undesired product formed by the parallel reaction

\section{REFERENCES}

Balakotaiah, V. and Luss, D., 1984, Global analysis of the multiplicity features of multi reaction lumped parameter systems. Chem. Enyng Sci. 39, 865-881.

Westerterp, K. R., 1962, Maximum allowable temperatures in chemical reactors. Chem. Engng Sci. 17, 423-433.

Westerterp, K. R. and Jansma, E., 1985, Safe design and operation of tank reactors for multiple reactions; uniqueness and multiplicity. Chem. Engng Sci. 40, 1469-1476.

Westerterp, K. R and Overtoom, R. R. M., 1985, Safe design of cooled tubular reactors for exothermic multiple reactions; consecutive reactions. Chem. Engng Sci. 40, 115-165.

Westerterp, K. R. and Ptasinsky, K. J., 1984a, Safe design of cooled tubular reactors for exothermic multiple reactions; parallel reactions. Development of criteria. Chem. Engng Sci. 39, 235-244.

Westerterp, K. R. and Ptasinsky, K. J., 1984b, Safe design of cooled tubular reactors for exothermic multiple reactions; parallel reactions. The design and operation of an ethylene oxide reactor. Chem. Engng Sci. 39, 245-252.

Westerterp, K. R., Ptasinsky, K. J. and Overtoom, R. R. M., $1984 a$, Safe design of cooled tubular reactors for exothermic multiple first order reactions. ACS Symp. Ser. No. 237, pp. 323-335.

Westerterp, K. R., van Swaaij, W. P. M. and Beenackers, A. A. C. M., 1984b, Chemical Reactor Design and Operation, pp. 305-307. John Wiley, Chichester.

Wohlfahrt, K. and Emig, G., 1980, Compare maleic anhydride routes. Hydrocarh. Process. 59(Iune), 83-90. 